\title{
Correção cirúrgica simultânea da fibrilação atrial e da insuficiência mitral em criança
}

\author{
Francisco GREGORI Jr. ${ }^{\star}$, Samuel Silva da SILVA*, Ulisses Alexandre CROTI ${ }^{\star \star}$, Sergio Shiguero \\ HAYASHI ${ }^{\star *}$, Icanor Antônio RIBEIRO**, Pedro Aloizio KRELING*
}

RBCCV 44205-299

Gregory Jr. F, Silva S S, Croti U A, Hayashi S S, Ribeiro I A, Kreling P A - Correção cirúrgica simultânea da fibrilação atrial e da insuficiência mitral em criança. Rev Bras Cir Cardiovasc 1996; 11 (2): 123-5.

RESUMO: Plastia valvar mitral combinada com cirurgia de COX (labirinto) foi realizada em uma paciente de 12 anos, para correção de insuficiência mitral reumática e fibrilação atrial crônica. Um ano após a cirurgia, a paciente encontra-se assintomática e em ritmo sinusal. $O$ ecodopplercardiograma mostrou redução importante do átrio esquerdo e o cateterismo, assim como o ecodopplercardiograma confirmaram sístole atrial efetiva.

DESCRITORES: Fibrilação atrial, cirurgia. Insuficiência da valva mitral, cirurgia. Cardiopatia reumática, cirurgia. Cox, operaçāo de.

\section{INTRODUÇÃO}

O tratamento cirúrgico da fibrilação atrial pela técnica de COX ("labirinto") tem apresentado resultados tardios bastante satisfatórios (1,2). A cirurgia conjuntiva para doença valvar mitral e fibrilação atrial crônica também tem mostrado evolução similar $(3,4)$. Realizamos a combinação destes procedimentos em 60 pacientes empregando-se a técnica clássica proposta inicialmente por Cox, exceto que a crioablação foi substituída por extensa dissecção do anel mitral e tricúspide (5). Em quase $90 \%$ dos casos,o ritmo pós-operatório passou a sinusal.

Destes, uma menina de 12 anos com insuficiência mitral reumática e fibrilação atrial crônica foi submetida a plástica valvar mitral e cirurgia de Cox I, sem crioablação. O resultado excelente e a ausência de descrição na literatura de tal procedimento em criança nos motivou à presente apresentação.

\section{RELATO DO CASO}

Uma menina de 12 anos de idade foi admitida no hospital apresentando insuficiência cardiaca em classe funcional IV (New York Heart Association), insuficiência mitral e fibrilação atrial com duração acima de um ano, apesar de adequado tratamento farmacológico. Sopro holosistólico $6 / 6$ estava presente no ápex, irradiando-se para a maior parte do hemitórax esquerdo. O eletrocardiograma mostrava fibrilação atrial e a radiografia de tórax uma importante cardiomegalia (Figura 1). O ecodopplercardiograma demonstrava regurgitação mitral importante e uma grande dilatação do átrio esquerdo $(7,6$ centímetros) (Figura 3).

A cirurgia foi indicada para correção da insuficiência mitral e fibrilação atrial pela técnica de Cox I, uma vez que a fibrilação atrial era crônica, apesar do tratamento clínico adequado e cardioversão elé-

Trabalho realizado no Serviço de Cirurgia Cardiaca de Londrina. Faculdade de Medicina da Universidade Estadual de Londrina - Hospital Evangélico. Londrina, PR, Brasil.

Recebido para publicação em março de 1996.

* Da Faculdade de Medicina da Universidade Estadual de Londrina e do Hospital Evangélico de Londrina.

** Do Hospital Evangélico de Londrina.

Endereço para correspondência: Francisco Gregori Jr. - Rua Paes Leme, 1264 - $7^{\circ}$ andar, sala 701. CEP: 86.010-520. Tel: (043) 324-5276 - Fax: (043) 324-5142 - Londrina, PR, Brasil. 
Gregory Jr. F, Silva S S, Croti U A, Hayashi S S, Ribeiro I A, Kreling P A - Correção cirúrgica simultânea da fibrilação atrial e da insuficiência mitral em criança. Rev Bras Cir Cardiovasc 1996; 11 (2): 123-5.

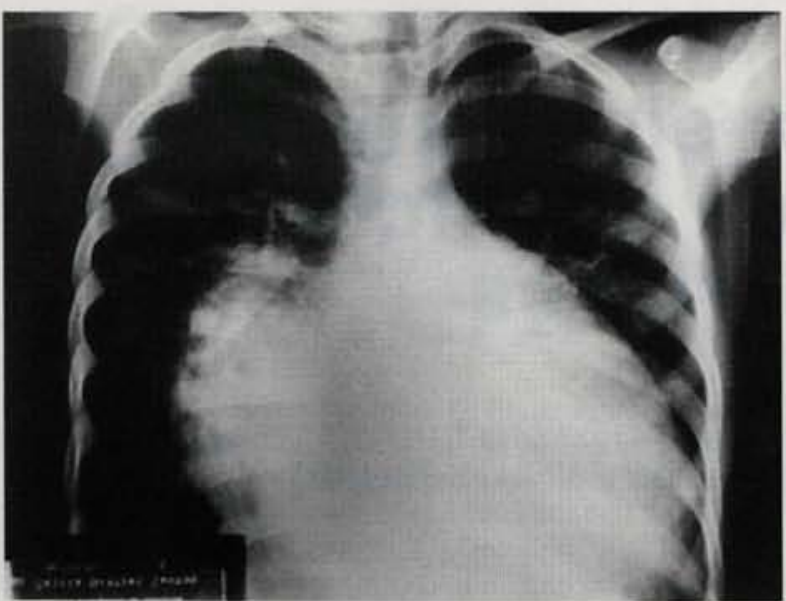

Fig. 1 - Radiografia de tórax (pré-operatório).

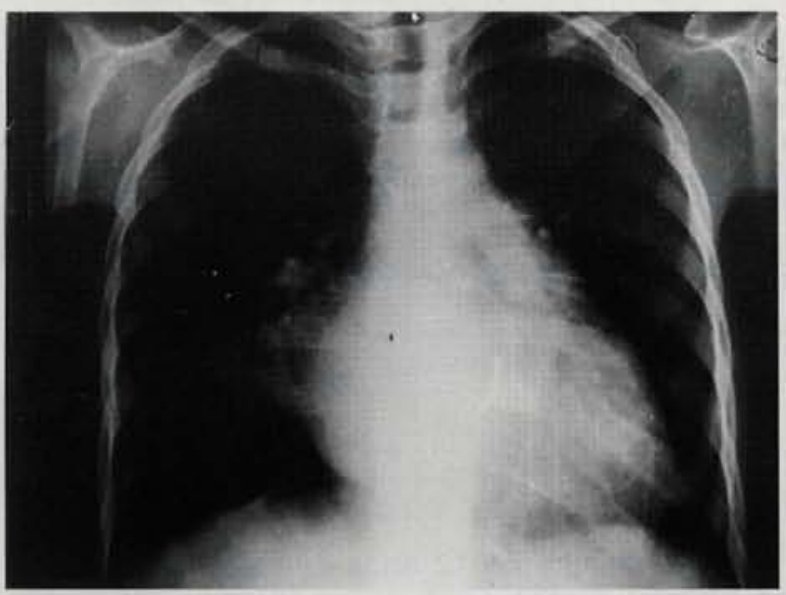

Fig. 2 - Radiografia de tórax (pós-operatório).

trica. Após o estabelecimento de circulação extracorpórea hipotérmica $\left(25^{\circ} \mathrm{C}\right)$, empregando-se cardioplegia cristalóide (St. Thomas), foi realizada a cirurgia clássica de Cox I. Os anéis mitral e tricúspide foram amplamente dissecados e as fibras miocárdicas seccionadas, não se utilizando a crioablação. A valva mitral, exposta, apresentava importante dilatação do anel mitral com alongamento da maioria das cordas tendineas. As cordas alongadas foram encurtadas ao nível do folheto e a dilatação anular corrigida com anel protético aberto (6). O tempo de anóxia do miocárdio foi de 80 minutos. A valva mitral ficou suficiente após o reparo e a paciente apresentou ritmo juncional durante 0 intra-operatório. O pós-operatório transcorreu sem complicações. Quatro dias após, o ritmo passou a sinusal e manteve-se até o décimo dia, quando um novo episódio de fibrilação atrial apareceu. Foi medicada com quinidina, e retornou o ritmo sinusal, mesmo após a suspensão da droga.
A paciente recebeu alta hospitalar em boas condições, tendo sido reavaliada 1 ano após a operação, estando assintomática, sem sopros e em ritmo sinusal sem medicação antiarrítmica. Foi submetida a exame radiológico de tórax, ecodopplercardiograma, Holter de 2 canais e cateterismo cardíaco. Houve importante redução da área cardiaca aos raios $X$ de tórax (Figura 2 ). $O$ diâmetro do átrio esquerdo medido pelo ecocardiograma era 5.0 centímetros e a valva mitral suficiente (Figura 4). O Holter mostrou ritmo sinusal constante e o cateterismo revelou a presença de contração atrial direita e esquerda efetiva e sincrônica (Onda A).

\section{COMENTÁRIOS .}

A insuficiência mitral em crianças é freqüente, especialmente devido a doença reumática $e$, rara-

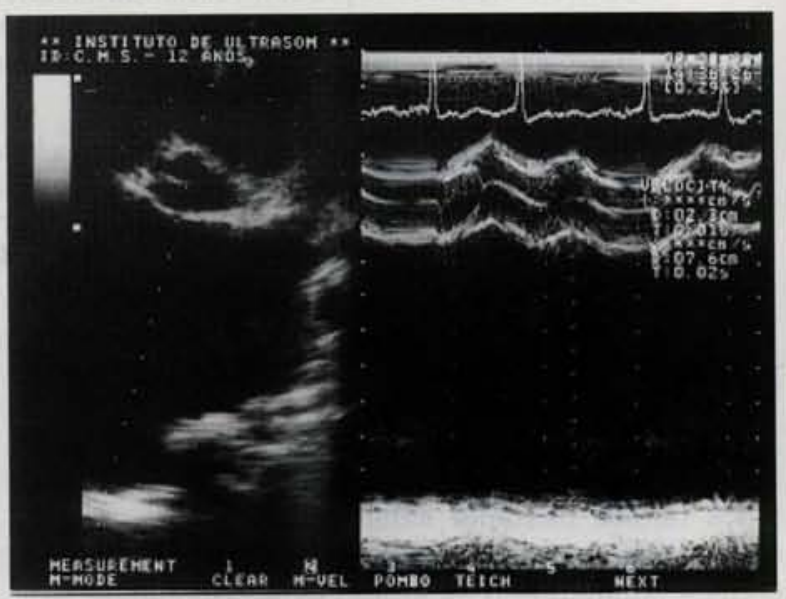

Fig. 3 - Ecodopplercardiograma pré-operatório. Observa-se grande aumento do átrio esquerdo ( 7,6 centimetros).

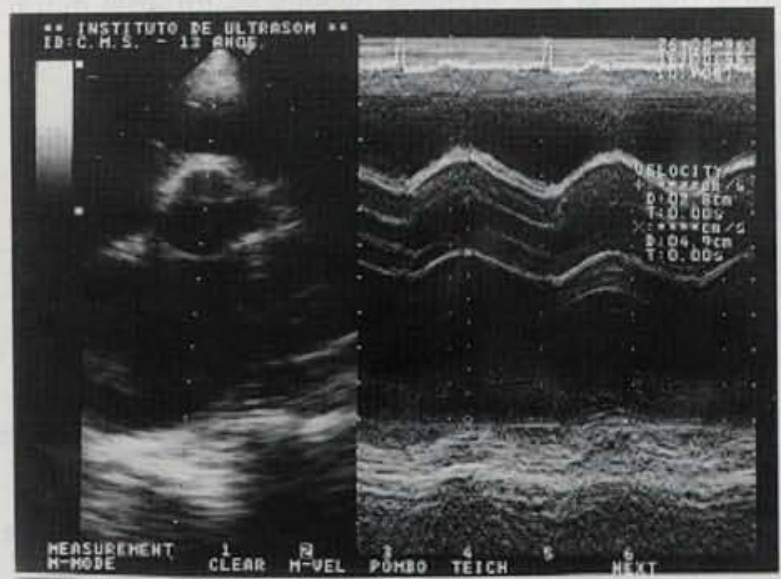

Fig. 4 - Ecodopplercardiograma (pós-operatório). Observa-se diminuição do diâmetro do átrio esquerdo ( 5,0 centimetros). 
Gregory Jr. F, Silva S S, Croti U A, Hayashi S S, Ribeiro I A, Kreling P A - Correçāo cirúrgica simultânea da fibrilaçāo atrial e da insuficiência mitral em criança. Rev Bras Cir Cardiovasc 1996; 11 (2): 123-5.

mente, as lesões são acompanhadas de fibrilação atrial.

Em nosso Serviço a cirurgia conservadora da valva mitral tem sido rotineira, principalmente em crianças e pacientes jovens. A fibrilação atrial tem, muitas vezes, persistido no pós-operatório tardio, apesar da diminuição do tamanho do átrio esquerdo.

Desde agosto de 1993, temos empregado a cirurgia de Cox I sem crioablação associada com correção da doença valvar mitral em casos com fibrilação atrial crônica que não respondem ao tratamento farmacológico. Em 60 casos operados foi observado que o ritmo em cerca de $90 \%$ reverteu para sinusal. Baseados nestes resultados satisfatórios, não temos mudado a técnica de Cox I para a recente modificação, Cox III. Achamos que esta técnica poderia ser utilizada em crianças com valvopatia mitral e fibrilação atrial crônica, o que ampliaria a indicação, já que, aproximadamente $30 \%$ dos nossos pacientes com problemas na valva mitral têm idade inferior a 16 anos. No caso descrito, a presença de um átrio esquerdo enorme tornou difícil a reversão da fibrilação atrial com o tratamento farmacológico e cardioversão elétrica, sendo indicada, portanto, a operação conjunta.

O excelente resultado obtido abre uma nova estratégia para o tratamento da valvopatia mitral associada com fibrilação atrial em crianças, especialmente em países onde a doença reumática é freqüente.

RBCCV 44205-299

Gregory Jr. F, Silva S S, Croti U A, Hayashi S S, Ribeiro I A, Kreling P A - Successful combined operation for mitral insufficiency and atrial fibrillation in a 12 year-old patient. Rev Bras Cir Cardiovasc 1996;11 (2): $123-5$.

ABSTRACT: A combined mitral reconstructive surgery and Cox-maze operation without cryoablação was performed in a 12-year-old female patient for the correction of a rheumatic mitral insufficiency and chronic atrial fibrillation. One year postoperative the patient was assymptomatic, in sinusal rhythm and no murmurs were observed. The Doppler echocardiogram showed an important reduction of the left atrial diameter and the cardiac catheterism confirmed an effective atrial systole.

DESCRIPTORS: Atrial fibrillation, surgery. Mitral valve insufficiency, surgery. Rheumatic heart disease, surgery. Cox operation.

\section{REFERÊNCIAS BIBLIOGRÁFICAS}

1 Cox J L, Boineau J P, Schuessler R B, Kater K M, Lappas D G - Five-year experience with the maze procedure for atrial fibrillation. Ann Thorac Surg 1994; 56: 814-24.

2 McCarthy P M, Castle L W, Maloney J D et al. - Initial experience with the maze procedure for atrial fibrillation. J Thorac Cardiovasc Surg 1993; 105: 1077-81.

Jatene A D, Sosa E, Tarasoutch F, Jatene M B, Pomerantzeff P M A - Tratamento cirúrgico da fibrilação arterial. Procedimento do "labirinto": experiência inicial. Rev Bras Cir Cardiovasc 1992; 7: 107-11.
4 Kosakai $Y$, Kawaguchi A T, Isobe $F$ et al - Cox-maze procedure for chronic atrial fibrillation associated with mitral valve disease. J Thorac cardiovasc Surg 1994; 108: 1049-55.

5 Gregory Jr. F, Cordeiro C O, Silva S S, Aquino W K, Nechar Jr. A - Cox-maze operation without cryoablation for the treatment of chronic atrial fibrillation. Ann Thorac Surg 1995; 60: 361-4.

6 Gregori Jr. F, Silva S S, Hayashi S S, Aquino W, Cordeiro C, Silva R - Mitral valvuloplasty with a new prosthetic ring: analysis of the first 105 cases. Eur J Cardio-thorac Surg 1994, 8: 168 72. 